\title{
Instabilities, turbulence, and mixing in the ocean of accreting neutron stars
}

\begin{abstract}
V. Urpin
Departament de Fisica Aplicada, Universitat d'Alacant, Ap. Correus 99, 03080 Alacant, Spain

A.F.Ioffe Institute for Physics and Technology and Isaak Newton Institute of Chili, Branch in St. Petersburg, 194021 St. Petersburg, Russia

e-mail: vadim.urpin@uv.es

Received 7 October 2004 / Accepted 17 March 2005

Abstract. We consider the stability properties of the ocean of accreting magnetic neutron stars. It turns out that the ocean is always unstable due to the combined influence of the temperature and chemical composition gradients along the surface and of the Hall effect. Both the oscillatory and non-oscillatory modes can be unstable in accreting stars. The oscillatory instability grows on a short timescale $\sim 0.1-10 \mathrm{~s}$ depending on the lengthscale of a surface inhomogeneity and the wavelength of perturbations. The instability of non-oscillatory modes is typically much slower and can develop on a timescale of hours or days. Instability generates a weak turbulence that can be responsible for mixing between the surface and deep ocean layers and for spreading the accreted material over the stellar surface. Spectral features of heavy elements can be detected in the atmospheres of accreting stars due to mixing, and these features should be different in neutron stars with both stable and unstable burning. Motions caused by instability can also be the reason for slow variations in the luminosity.
\end{abstract}

Key words. magnetohydrodynamics (MHD) - convection - stars: neutron - stars: magnetic fields

\section{Introduction}

The surface layers of accreting neutron stars are in a melted state because of the heat production due to nuclear burning of the accreted material. The way a neutron star burns the accreted hydrogen and helium to heavier elements is sensitive to the accretion rate (e.g., Bildsten 1998). This nuclear burning is very to be stable for relatively high accretion rate, $\dot{M} \geq 2.6 \times$ $10^{-8} M_{\odot} \mathrm{yr}^{-1}$. Burning at such accretion rates is not hot enough to produce iron group or heavier elements; thus the surface layers of neutron stars consist mainly of CNO group elements (Brown \& Bildsten 1998). Fusion of this fuel to heavier elements does not occur at the density $\rho \leq 10^{9} \mathrm{~g} \mathrm{~cm}^{-3}$. If the neutron star is accreting pure He then the burning at $\rho>10^{9} \mathrm{~g} \mathrm{~cm}^{-3}$ produces iron group elements, but if the star accretes a mixture of $\mathrm{H}$ and $\mathrm{He}$ then the products are much havier (Schatz et al. 1999). The low ionic charge and a relatively high temperature $\left(\geq 10^{8} \mathrm{~K}\right)$ caused by the nuclear burning delay cristallization until very high densities are reached. Therefore neutron stars accreting at a high accretion rate are covered with massive oceans. Neutron stars accreting at lower rates, $\dot{M}<2.6 \times$ $10^{-8} M_{\odot} \mathrm{yr}^{-1}$, burn accreted $\mathrm{H}$ and $\mathrm{He}$ directly onto iron group elements (Lewin et al. 1992; Schatz et al. 2001; Woosley et al. 2004), while the Coulomb interaction between heavy ions leads to crystallization at much lower densities and to a much shallow ocean.

MHD processes in the ocean are of general interest to studies of magnetic and thermal evolution, thermonuclear burning, neutron star seismology, and properties of quasi-periodic oscillations. For instance, in weakly magnetized accreting neutron stars, the ocean of light elements supports shallow water waves, which can be the reason for low frequency $(\sim 5-7 \mathrm{~Hz})$ quasiperiodic oscillations in the brightest X-ray sources (Bildsten \& Cutler 1995; Bildsten et al. 1996). Under certain conditions, large-scale flows in the ocean can influence the magnetic evolution of accreting neutron stars (Bisnovatyi-Kogan \& Komberg 1974; Rai Choudhuri \& Konar 2002). MHD phenomena can play a particular role during thermonuclear X-ray bursts. Thus, if an unstable nuclear burning is not spherically symmetric, then burning fronts can spread around the star by igniting the accumulated cold fuel ahead of the moving front (Fryxell \& Woosley 1982; Bildsten 1993; Spitkovsky et al. 2001). The propagation speed can be considerably increased when convection and enhanced mixing are occurring at the burning front since convective motions are very efficient at transporting heat in the ocean. Note that effects caused by rotation should be important during X-ray bursts since most bursters are rapidly rotating. Most likely, an expanding ocean rotates differentially, and differential rotation can be the reason for MHD instabilities (Cumming \& Bildsten 2000; Menou 2004).

Mixing in stars is often atributed to hydrodynamic instabilities, such as convection. Standard thermal convection arises if the temperature gradient is superadiabatic and occurs only in the atmosphere of hot NSs with a weak magnetic field (Miralles et al. 1997). Convection, however, is not the only instability that occurs in the ocean. Stability properties of accreting neutron 
stars can be complicated because of the presence of a strong magnetic field, $B \sim 10^{12}-10^{13} \mathrm{G}$, and of the thermal and compositional gradients that generally are not parallel to gravity $\boldsymbol{g}$. A possible occurrence of the Kruskal-Schwarzschild instability in polar caps of accreting neutron stars has been considered by Litwin et al. (2001), who found that ballooning modes with the displacement perpendicular to the magnetic field can be unstable if the overpressure at the bottom of the neutron star ocean exceeds magnetic pressure by a factor $\sim 8 a / h$, where $a$ and $h$ are the horizontal lengthscale of the cap and the density scale hight, respectively. The crucial point of the instability is linetying to the neutron star crust and, therefore, unstable modes are localized to within a density scale height of the ocean bottom. The ballooning instability can arise on a timescale $\sim 30 \mathrm{~h}$ if $\dot{M} \sim 10^{-10} M_{\odot} /$ yr and, most likely does not affect the nuclear burning but can degrade the confinement to prevent accumulation of mass above the instability threshold.

One more instability that can manifest itself in accreting neutron stars is the well-known Parker instability (Parker 1966). The downward flow of accreted matter may generate strong non-uniformity in the magnetic field and, hence, the electric current, which in turn can lead to Parker-type instability (Cumming et al. 2001). Note that this instability arises only if both the magnetic field and its non-uniformities are sufficiently strong and if the field decreases with height. Employing the effect of thermal diffusivity, Cumming et al. (2001) estimated that the critical field above which the instability occurs is $\sim 10^{12} \mathrm{G}$ at the top of the crust. The Parker-type instability can be of particular importance if the magnetic field in the surface layers has a complex geometry, for example, such as one considered by Urpin \& Gil (2004).

The material accreted from a companion star carries angular momentum. The inflow of angular momentum promotes differential rotation in the ocean of neutron stars and can cause rotation induced instabilities. These instabilities occur when the angular velocity gradient is so steep that the destabilizing effect of shear overwhelms the stabilizing effect of buoyancy (see, e.g., Zahn 1983). In the context of neutron star oceans, the instabilities caused by differential rotation have been considered by Fujimoto $(1988,1993)$. Turbulent motions generated by the instabilities transport the angular momentum in the ocean effectively, but the elemental mixing is much less efficient because it is hampered by a stable stratification. Note that a stable stratification can suppress some rotation induced instabilities since the Brunt-Väisälä frequency is typically much larger than the angular velocity in accreting neutron stars (Cumming $\&$ Bildsten 2000). If differential rotation departs from the cylindrical symmetry, then the ocean can be subject to baroclinic instability (Cumming \& Bildsten 2000). A stable stratification generally suppresses the modes with horizontal lengthscales that are much longer than a pressure scale height, but shortwavelength can be unstable, and turbulent transport caused by the baroclinic instability can be significant during type $1 \mathrm{X}$-ray bursts. Note also that some mixing in accreting neutron stars can be provided by tidal waves (Lou 2001). It is unlikely, however, that this mechanism is efficient since tidal waves in the neutron star ocean shoud have a small amplitude because of high gravity.
In the present paper, we consider the instability caused by horizontal inhomogeneities of the temperature and chemical composition in the ocean of accreting magnetic neutron stars. The thermal and compositional state of the ocean departs substantially from the spherical symmetry for such stars. The departure can be caused, for example, by the accreted hydrogen and helium focusing onto the magnetic poles. It is usually assumed that the accretion flow interacts with the magnetosphere at the so-called Alfvén radius, $R_{\mathrm{A}}$, which is determined by the balance of the magnetic and inertial forces. For the standard neutron star with a dipole magnetic field and with mass $M=1.4 M_{\odot}$ and radius $R \approx 10^{6} \mathrm{~cm}$, the Alfvén radius is given by

$R_{\mathrm{A}} \sim 2 \times 10^{9} B_{13}^{4 / 7} \dot{M}_{-10}^{-2 / 7} \mathrm{~cm}$,

where $B_{13}=B / 10^{13} \mathrm{G}$ and $\dot{M}_{-10}=\dot{M} /\left(10^{-10} M_{\odot} \mathrm{yr}^{-1}\right)$. For a weakly magnetized star accreting at a high accretion rate, the Alfvén radius becomes comparable to the stellar radius, and the magnetic field cannot funnel the accreted material onto the magnetic poles. Therefore, the distribution of the accreted material over the stellar surface is more or less smoothed in such stars. In contrast, a strongly magnetized neutron star accretes the material predominantly onto the magnetic poles, and distribution of the chemical composition and temperature is substantially non-spherical in the ocean of magnetized stars (Brown \& Bildsten 1998).

It has been shown by Urpin (2004) that the combined influence of the Hall effect and a thermal gradient perpendicular to $\boldsymbol{g}$ can be responsible for instability in the ocean of isolated neutron stars. In accreting neutron stars, the presence of a compositional gradient that generally is also non-parallel to $\boldsymbol{g}$ can lead to similar instability. This sort of instability is qualitatively different from instabilities already considered in accreting neutron stars because it occurs only in the presence of the Hall effect. The criterion of instability can be satisfied in many accreting neutron stars if the compositional gradient is not parallel to gravity.

This paper is organized as follows. In Sect. 2, we consider the main equations governing hydrodynamic motions in the ocean of accreting magnetic neutron stars and derive the dispersion equation. Section 3 deals with the criterion and growth time of instability. In Sect. 4, we discuss mixing processes associated to compositional instability in the ocean.

\section{The basic equations}

We consider the linear stability of the ocean on an accreting neutron star assuming that both the temperature and chemical composition can generally depart from spherical symmetry in the unperturbed state. We do not specify the mechanisms responsible for these departures but they can originate, for example, in the magnetic field $\boldsymbol{B}$ that funnels the accretion flow onto the poles and provides anisotropy to the heat transport. As a result, gradients of the temperature and chemical composition are not parallel to gravity $\boldsymbol{g}$, which is approximately radial in the ocean.

The depth of the ocean depends crucially on the temperature and chemical composition and varies over a wide range. 
Crystallization of a Coulomb liquid occurs when the ion coupling parameter $\Gamma=Z^{2} e^{2} /\left(a k_{\mathrm{B}} T\right)$ reaches the critical value $\Gamma=\Gamma_{\mathrm{m}} \approx 170-180$ (Slattery et al. 1980; Potekhin \& Chabrier 2000); $a=\left(3 / 4 \pi n_{i}\right)^{1 / 3}$ is the mean inter-ion distance, $n_{i}$ and $Z$ are the number density and the charge number of ions, respectively; $k_{\mathrm{B}}$ is the Boltzmann constant. Then, the crystallization temperature is

$T_{\mathrm{m}}=\frac{Z^{2} e^{2}}{a k_{\mathrm{B}} \Gamma_{\mathrm{m}}} \approx 1.3 \times 10^{5} Z^{5 / 3} x^{1 / 3}\left(\frac{170}{\Gamma_{\mathrm{m}}}\right) \mathrm{K}$,

where $x=Z \rho / 10^{6} A \mathrm{~g} / \mathrm{cm}^{3}$, and $A$ is the atomic number of ions. In neutron stars with temperature $T \geq 10^{8}$ which is more or less typical for stars accreting with the rate $\sim 10^{-9} M_{\odot} \mathrm{yr}^{-1}$, crystallization occurs at the density $\rho \sim 3 \times 10^{10} \mathrm{~g} / \mathrm{cm}^{3}$ if $Z=8$.

The equations governing velocity, magnetic field, and thermal balance are (see, e.g., Landau \& Lifshitz 1959)

$\dot{\boldsymbol{v}}+(\boldsymbol{v} \cdot \nabla) \boldsymbol{v}=-\frac{\nabla p}{\rho}+\boldsymbol{g}+\frac{1}{4 \pi \rho}(\nabla \times \boldsymbol{B}) \times \boldsymbol{B}$,

$\dot{\rho}+\nabla \cdot(\rho \boldsymbol{v})=0$,

$\dot{\boldsymbol{B}}-\nabla \times(\boldsymbol{v} \times \boldsymbol{B})=-\nabla \times[\hat{\eta} \cdot(\nabla \times \boldsymbol{B})]$,

$\nabla \cdot \boldsymbol{B}=0$,

$\dot{T}-\frac{\beta}{\rho c_{\mathrm{p}}} \dot{p}+\boldsymbol{v} \cdot(\Delta \nabla T)=\frac{1}{\rho c_{\mathrm{p}}} \nabla \cdot(\hat{\kappa} \cdot \nabla T)$,

where $v$ is the fluid velocity; $\Delta \nabla T=\nabla T-\nabla_{\mathrm{ad}} T$ is the difference between the real and adiabatic temperature gradients, $\nabla_{\mathrm{ad}} T=\beta \nabla p / \rho c_{\mathrm{p}} ; \beta=-(\partial \ln \rho / \partial \ln T)_{\mathrm{p}}$ is the thermal expansion coefficient and $c_{\mathrm{p}}$ the specific heat at constant pressure, $p$; and finally $\hat{\eta}=c^{2} \hat{R} / 4 \pi, \hat{R}$ and $\hat{\kappa}$ are tensors of the electrical resistivity and thermal conductivity. Following some tensor operations on Eqs. (5) and (7), we have

$\hat{\kappa} \cdot \nabla T=\kappa^{(0)} \boldsymbol{b}(\boldsymbol{b} \cdot \nabla T)+\kappa^{(B)}[\nabla T-\boldsymbol{b}(\boldsymbol{b} \cdot \nabla T)]+\kappa^{(\wedge)} \boldsymbol{b} \times \nabla T$,

where $\kappa^{(0)}$ and $\kappa^{(B)}$ are the tensor components along and across the magnetic field, respectively, $\kappa^{(\wedge)}$ is the so called Hall component, and $\boldsymbol{b}=\boldsymbol{B} / B$. An analogous expression can be written for $\hat{\eta}$. Note that for the magnetic diffusivity we have $\eta^{(0)}=$ $\eta^{(B)}=\eta^{(\wedge)} / \alpha$ where $\alpha$ is the Hall parameter,

$\alpha=\Omega_{\mathrm{Be}} \tau \approx \frac{9.9 \times 10^{3} B_{13}}{Z \Lambda\left(1+x^{2 / 3}\right)}$,

$\Omega_{\mathrm{Be}}$ and $\tau$ are the electron gyrofrequency and relaxation time, respectively; and $\Lambda$ is the Coulomb logarithm.

We neglect the viscous term in the momentum Eq. (3) since viscosity plays a less important role among kinetic processes in the ocean. Usually, both electrons and ions contribute to the shear viscosity. The ion shear viscosity dominates at a relatively low density but is typically much smaller than magnetic diffusivity $\eta$ and thermal diffusivity, $\chi=\kappa / \rho c_{\mathrm{p}}$. Electron viscosity is greater in deep layers of the ocean (Itoh et al. 1987). The ratio of electron viscosity $v_{\mathrm{e}}$ and magnetic diffusivity along the magnetic field is given by

$\frac{v_{\mathrm{e}}}{\eta^{(0)}}=\frac{2.2 \times 10^{2}}{A Z \Lambda} \frac{x^{5 / 3}}{\left(1+x^{2 / 3}\right)^{2}}$, where $\Lambda$ is the Coulomb logarithm. The kinematic viscosity becomes comparable to the magnetic one only at a high density $\geq 10^{9} \mathrm{~g} / \mathrm{cm}^{3}$ if the ocean consists of light elements. Therefore, neglecting viscosity in Eq. (3) seems to be qualitatively justified.

We assume that the ocean is in hydrostatic equilibrium in the unperturbed state,

$\frac{\nabla p}{\rho}=\boldsymbol{G}=\boldsymbol{g}+\frac{1}{4 \pi \rho}(\nabla \times \boldsymbol{B}) \times \boldsymbol{B}$.

Taking the curl of this equation, we have

$\nabla \rho \times \boldsymbol{G}=-\frac{1}{4 \pi} \nabla \times[(\nabla \times \boldsymbol{B}) \times \boldsymbol{B}]$.

The density and, hence, temperature and compositional gradients have components perpendicular to $\boldsymbol{G}$ if the magnetic field is not force-free in the ocean.

Consider only relatively fast hydrodynamic processes varying on a timescale shorter than the characteristic timescale of nuclear reactions. The time to burn all hydrogen into CNO-group elements is, for example, on the order of $20 \mathrm{~h}$ at high accretion rates (see, e.g., Cumming \& Bildsten 2000). Under this assumption, the number density of a species $j$ satisfies the continuity equation

$\frac{\partial n_{j}}{\partial t}+\nabla \cdot\left(n_{j} \boldsymbol{v}\right)=0$

For our purposes, it will be convenient to characterize the composition by a mass fraction of species $X_{j}=\rho_{j} / \rho$, where $\rho_{j}$ is the density of species $j$. Note that if the number of species is $N$ then only $N-1$ fractions $X_{j}$ are linearly independent, since $X_{j}$ satisfy the condition $\sum_{N} X_{j}=1$; summation is over all species of ions. If the chemical composition is frozen in a moving fluid parcel, then the equations governing $X_{j}$ read

$\frac{\partial X_{j}}{\partial t}+\nabla \cdot\left(X_{j} v\right)=0$

The equations for small perturbations can be obtained by linearization of Eqs. (3)-(7), and (13). We assume that in the unperturbed state, a steady temperature and composition gradients are maintained, and there are no motions. In what follows, small perturbations will be marked by the subscript 1 , but subscripts will be omitted for unperturbed quantities. We consider short-wavelength perturbations with spatial and temporal dependence $\exp (\gamma t-\mathrm{i} \boldsymbol{k} \cdot \boldsymbol{r})$ where $\boldsymbol{k}$ is the wave-vector. Linearization of Eqs. (3)-(7), (13) yields

$\gamma \boldsymbol{v}_{1}=\mathrm{i} \boldsymbol{k} \frac{p_{1}}{\rho}+\boldsymbol{G} \frac{\rho_{1}}{\rho}-\frac{\mathrm{i}}{4 \pi \rho}\left(\boldsymbol{k} \times \boldsymbol{B}_{1}\right) \times \boldsymbol{B}$,

$\gamma \rho_{1}+\boldsymbol{k} \cdot \boldsymbol{v}_{1}=0$,

$\left(\gamma+\omega_{\eta}\right) \boldsymbol{B}_{1}=-\mathrm{i}(\boldsymbol{k} \cdot \boldsymbol{B}) \boldsymbol{v}_{1}-\eta_{\wedge}(\boldsymbol{k} \cdot \boldsymbol{b}) \boldsymbol{k} \times \boldsymbol{B}_{1}$,

$\boldsymbol{k} \cdot \boldsymbol{B}_{1}=0$

$\left(\gamma+\omega_{\kappa}\right) T_{1}-\frac{\gamma \beta}{\rho c_{\mathrm{p}}} p_{1}=-\boldsymbol{v}_{1} \cdot(\Delta \nabla T)$,

$\gamma X_{1 j}+\boldsymbol{v}_{1} \cdot \nabla X_{j}=0$, 
where the characteristic inverse timescales of the ohmic dissipation and thermal diffusion are given by $\omega_{\eta}=\eta^{(0)} k^{2}$ and $\omega_{\kappa}=\left[\kappa^{(B)} k^{2}+\left(\kappa^{(0)}-\kappa^{(B)}\right)(\boldsymbol{k} \cdot \boldsymbol{b})^{2}\right] / \rho c_{\mathrm{p}}$, respectively.

Perturbations of the pressure in Eq. (14) can be expressed in terms of perturbations of the density, temperature, and fractions of species $X_{1 j}$, using the equation of state,

$$
\frac{p_{1}}{p}=\left(\frac{\partial \ln p}{\partial \ln \rho}\right)_{\mathrm{T}, \mathrm{Y}}\left(\frac{\rho_{1}}{\rho}+\beta \frac{T_{1}}{T}+\sum^{N-1} \delta_{j} X_{1 j}\right),
$$

where $\delta_{j}=-\left(\partial \ln \rho / \partial X_{j}\right)_{\mathrm{pT}}$ are the coefficients of chemical expansion, and summation is over $N-1$ linearly independent species.

Consider Eqs. (14)-(20) in the case $k c_{\mathrm{s}} \gg \gamma$ that corresponds to the Boussinesq approximation for slowly varying modes. Then, the dispersion equation reads

$\gamma^{6}+a_{5} \gamma^{5}+a_{4} \gamma^{4}+a_{3} \gamma^{3}+a_{2} \gamma^{2}+a_{1} \gamma+a_{0}=0$

where

$$
\begin{aligned}
& a_{5}=\omega_{\kappa}+2 \omega_{\eta}, \\
& a_{4}=\omega_{\eta}^{2}+\omega_{\wedge}^{2}+2 \omega_{\eta} \omega_{\kappa}+2 \omega_{\mathrm{A}}^{2}-\omega_{0}^{2}, \\
& a_{3}=\omega_{\kappa}\left(\omega_{\eta}^{2}+\omega_{\wedge}^{2}+2 \omega_{\mathrm{A}}^{2}-\omega_{\mathrm{X}}^{2}\right)+2 \omega_{\eta}\left(\omega_{\mathrm{A}}^{2}-\omega_{0}^{2}\right), \\
& a_{2}=2 \omega_{\eta} \omega_{\kappa}\left(\omega_{\mathrm{A}}^{2}-\omega_{\mathrm{X}}^{2}\right)-\omega_{0}^{2}\left(\omega_{\eta}^{2}+\omega_{\wedge}^{2}+\omega_{\mathrm{A}}^{2}\right)+\omega_{\mathrm{A}}^{4}, \\
& a_{1}=\omega_{\mathrm{A}}^{2}\left[\omega_{\kappa} \omega_{\mathrm{A}}^{2}-\omega_{\eta}\left(\omega_{0}^{2}+\omega_{\mathrm{H}}^{2}\right]-\omega_{\kappa} \omega_{Y}^{2}\left(\omega_{\eta}^{2}+\omega_{\wedge}^{2}+\omega_{\mathrm{A}}^{2}\right),\right. \\
& a_{0}=-\omega_{\kappa} \omega_{\eta} \omega_{\mathrm{A}}^{2}\left(\omega_{\mathrm{X}}^{2}+\omega_{\mathrm{XH}}^{2}\right) .
\end{aligned}
$$

The characteristic frequencies in this equation are

$\omega_{\wedge}=\alpha \eta_{\|} k(\boldsymbol{k} \cdot \boldsymbol{b}), \quad \omega_{\mathrm{A}}=\frac{(\boldsymbol{k} \cdot \boldsymbol{B})}{\sqrt{4 \pi \rho}}, \quad \omega_{\mathrm{X}}^{2}=\boldsymbol{D} \cdot \nabla X$,

$\omega_{\mathrm{g}}^{2}=\frac{\beta}{T} \boldsymbol{D} \cdot \Delta \nabla T, \quad \omega_{0}^{2}=\omega_{\mathrm{g}}^{2}+\omega_{\mathrm{X}}^{2}=\beta \boldsymbol{D} \cdot \boldsymbol{C}$,

$\omega_{\mathrm{H}}^{2}=\omega_{\mathrm{gH}}^{2}+\omega_{\mathrm{XH}}^{2}, \omega_{\mathrm{gH}}^{2}=\frac{\alpha \beta(\boldsymbol{k} \cdot \boldsymbol{b})}{k^{2} T} \Delta \nabla T \cdot(\boldsymbol{k} \times \boldsymbol{D})$,

$\omega_{\mathrm{XH}}^{2}=\frac{\alpha(\boldsymbol{k} \cdot \boldsymbol{b})}{k^{2}}(\boldsymbol{k} \times \boldsymbol{D}) \cdot \nabla X$,

where vectors $\boldsymbol{D}$ and $\boldsymbol{C}$ are given by

$\boldsymbol{D}=\boldsymbol{G}-\frac{\boldsymbol{k}}{k^{2}}(\boldsymbol{k} \cdot \boldsymbol{G}), \quad \boldsymbol{C}=\frac{\Delta \nabla T}{T}+\frac{\nabla X}{\beta}$,

and we denote $\nabla X=\sum^{N-1} \delta_{j} \nabla X_{j}$. In the limit $\omega_{\mathrm{X}} \approx \omega_{\mathrm{XH}} \approx 0$, Eq. (21) yields the dispersion equation for a chemically homogeneous ocean derived by Urpin (2004).

\section{The growth rate and criterion of instability}

Equation (21) describes six modes that exist in a chemically inhomogeneous ocean of magnetic neutron stars. Stability properties of the modes depend crucially on $\boldsymbol{k}$ and, under certain conditions, modes can be unstable. The dissipative frequences $\omega_{\eta}, \omega_{\wedge}$, and $\omega_{\kappa}$ are typically smaller than the dynamical frequences $\omega_{\mathrm{A}}, \omega_{0}$, and $\omega_{\mathrm{H}}$ except perturbations with a very short wavelength, $\lambda=2 \pi / k$. Therefore, the dispersion relations for some modes can be obtained by making use of the perturbation method and assuming that dissipative effects are small. We expand the growth rate as $\gamma=\gamma^{(0)}+\gamma^{(1)}+\gamma^{(2)}+\ldots$ where $\gamma^{(0)}, \gamma^{(1)}$, and $\gamma^{(2)}$ are terms of the zeroth, first, and second order in dissipative frequencies, respectively. The corresponding expansion should be made for the coefficients of Eq. (21) as well. In the zeroth order when dissipation is neglected, Eq. (21) reduces to a quadratic equation,

$\gamma^{(0) 4}+\left(2 \omega_{\mathrm{A}}^{2}-\omega_{0}^{2}\right) \gamma^{(0) 2}+\omega_{\mathrm{A}}^{2}\left(\omega_{\mathrm{A}}^{2}-\omega_{0}^{2}\right)=0$.

This equation describes four modes with the frequencies

$\gamma_{1,2}^{(0) 2}=-\omega_{\mathrm{A}}^{2}, \quad \gamma_{3,4}^{(0) 2}=-\omega_{\mathrm{A}}^{2}+\omega_{0}^{2}$.

The fifth and sixth roots of Eq. (21) are vanishing at the zeroth order of approximation, $\gamma_{5}^{(0)}=\gamma_{6}^{(0)}=0$. Usually, $\gamma^{(0) 2}<0$ for the modes 1-4 because the inequality $\omega_{0}^{2}>\omega_{\mathrm{A}}^{2}$ requires temperature and composition gradients that do not exist in neutron stars. Therefore, modes 1-4 are oscillatory, and their instability does not occur at the zeroth order.

For applicability of the perturbation procedure, the first corrections to $\gamma^{(0)}$ caused by dissipative effects have to satisfy the condition $\left|\gamma^{(1)}\right| \ll\left|\gamma^{(0)}\right| \sim \omega_{\mathrm{A}}$, since $\omega_{\mathrm{A}}$ is usually larger than other frequencies. The real parts of $\gamma^{(1)}$ for the modes 1-4 have a particularly simple form if $\left|\omega_{0}\right|$ and $\left|\omega_{\mathrm{H}}\right|$ satisfy the inequalities

$\left|\omega_{0}^{2}\right| \gg \omega_{\eta} \omega_{\kappa}\left(1+\alpha^{2}\right), \quad\left|\omega_{\mathrm{H}}^{2}\right| \gg \omega_{\eta} \omega_{\kappa}\left(1+\alpha^{2}\right)$.

Under this condition, the real parts of $\gamma^{(1)}$ are given by

$\operatorname{Re} \gamma_{1,2}^{(1)}=\frac{\omega_{\eta}\left(\omega_{\mathrm{H}}^{2}-\omega_{0}^{2}\right)}{2 \omega_{0}^{2}}$,

$\operatorname{Re} \gamma_{3,4}^{(1)}=-\frac{\omega_{\eta} \omega_{\mathrm{A}}^{2}\left[\omega_{\mathrm{H}}^{2}+\omega_{0}^{2}(1-\zeta)\right]}{2 \omega_{0}^{2}\left(\omega_{\mathrm{A}}^{2}-\omega_{0}^{2}\right)}$,

where $\zeta=\omega_{\kappa} \omega_{0}^{2} / \omega_{\eta} \omega_{\mathrm{A}}^{2}$. Note that if condition (24) is not fulfilled and dissipative effects dominate the dynamical influence of temperature and composition gradients along the surface, then modes $1-4$ are stable.

The roots 5 and 6 of Eq. (21) describe a couple of nonoscillatory modes and are linear in dissipative effects. To calculate these roots we should keep the terms in Eq. (21) up to the second order in dissipative frequencies assuming that $\gamma$ is linear at these frequencies. Then, roots 5 and 6 are approximately

$\gamma_{5,6}^{(1)} \approx-\frac{A_{1}}{2} \pm \sqrt{\frac{A_{1}^{2}}{4}-A_{0}}$,

where

$A_{0}=-\frac{\omega_{\kappa} \omega_{\eta}\left(\omega_{\mathrm{X}}^{2}+\omega_{\mathrm{XH}}^{2}\right)}{\omega_{\mathrm{A}}^{2}-\omega_{0}^{2}}$,

$A_{1}=\frac{\omega_{\kappa}\left(\omega_{\mathrm{A}}^{2}-\omega_{\mathrm{X}}^{2}\right)-\omega_{\eta}\left(\omega_{0}^{2}+\omega_{\mathrm{H}}^{2}\right)}{\omega_{\mathrm{A}}^{2}-\omega_{0}^{2}}$.

Modes 5 and 6 are secular and appear only due to dissipative effects. Note that contrary to modes $1-4$, expression (27) for nonoscillatory modes applies even for small wavelengths which do not satisfy the inequality (24). The only condition for applying Eq. (27) is $\left|\gamma_{5,6}^{(1)}\right| \ll \omega_{\mathrm{A}}$. 


\subsection{Instability of the oscillatory modes}

The real parts of oscillatory modes are given by Eqs. (25) and (26). Note that the vertical component of $\nabla X$ can be rather large because the chemical composition varies substantially with the ocean depth. Therefore, the contribution of $\nabla X$ to $\omega_{0}^{2}$ can usually be comparable to (or even larger than) that of $\Delta \nabla T$. As mentioned, usually $\omega_{\mathrm{A}}^{2}>\omega_{0}^{2}$ (see Eq. (23)). If $\omega_{0}^{2}>0$ then instability of oscillatory modes occurs if

$\omega_{\mathrm{H}}^{2}>\omega_{0}^{2}$ or $\omega_{\mathrm{H}}^{2}<\omega_{0}^{2}(\zeta-1)$.

In contrast, if stratification is convectively stable and $\omega_{0}^{2}<0$ then these modes are unstable if

$\omega_{\mathrm{H}}^{2}<\omega_{0}^{2}$ or $\omega_{\mathrm{H}}^{2}>\omega_{0}^{2}(\zeta-1)$.

In the particular case when gradients of the temperature and chemical composition are parallel to $\boldsymbol{G}$ and $\omega_{\mathrm{gH}}^{2}=\omega_{\mathrm{XH}}^{2}=0$, modes 1 and 2 are stable but modes 3 and 4 can be unstable if $\zeta>1$, or

$\omega_{\kappa} \omega_{0}^{2}>\omega_{\eta} \omega_{\mathrm{A}}^{2}$.

This condition generalizes the well-known criterion of oscillatory convection (see, e.g., Chandrasekhar 1961) for the case of a chemically inhomogeneous fluid. In the ocean of neutron stars, parameter $\zeta$ can be estimated as

$\zeta \sim 3 \times 10^{-3} \frac{T_{7} \lambda_{2}^{2}}{Z \Lambda^{2} B_{13}^{2} H_{3}} \frac{x^{2}\left[1+\left(1+x^{2 / 3}\right)^{1 / 2}\right]}{\left(1+x^{2 / 3}\right)^{2}}$,

where $H=C_{\|}^{-1}=\left(H_{\mathrm{T}}^{-1}+H_{\mathrm{X}}^{-1}\right)^{-1}, H_{\mathrm{T}} \equiv\left|(\Delta \nabla T / T)_{\|}^{-1}\right|$, and $H_{\mathrm{X}}=$ $\beta\left|(\nabla X)_{\|}\right|^{-1}$ are the lengthscales of the temperature and chemical composition along $\boldsymbol{G}$ (the subscript $\|$ denotes the component parallel to $\boldsymbol{G}), H_{3}=H / 10^{3} \mathrm{~cm}$; and $\lambda_{2}=\lambda / 100 \mathrm{~cm}$. Usually $\zeta<1$, and condition (30) is not satisfied except for very hot stars with a low magnetic field (Miralles et al. 1997).

If $|\zeta| \ll 1$, then conditions (28) and (29) read

$\left|\omega_{\mathrm{H}}^{2}\right|>\left|\omega_{0}^{2}\right|$.

This inequality can always be satisfied by a corresponding choice of $\boldsymbol{k}$ once $(\Delta \nabla T)_{\perp} \neq 0$ or $(\nabla X)_{\perp} \neq 0$, where $\perp$ denotes the component perpendicular to $\boldsymbol{G}$. Introducing local coordinates with the $z$-axis antiparallel to $\boldsymbol{G}$ and the $x$-axis aligned with $\boldsymbol{k}_{\perp}$, we have $\boldsymbol{G}=-G \boldsymbol{e}_{z}, \boldsymbol{k}=k_{x} \boldsymbol{e}_{x}+k_{z} \boldsymbol{e}_{z} ; \boldsymbol{e}_{x}, \boldsymbol{e}_{y}$, and $\boldsymbol{e}_{z}$ are unit vectors. The expressions for $\omega_{0}^{2}$ and $\omega_{\mathrm{H}}^{2}$ read in these coordinates

$\omega_{0}^{2}=\frac{k_{x}}{k^{2}} \beta G\left(k_{z} C_{x}-k_{x} C_{z}\right), \quad \omega_{\mathrm{H}}^{2}=\frac{k_{x}}{k^{2}} \alpha \beta G(\boldsymbol{k} \cdot \boldsymbol{b}) C_{y}$,

where $C_{x, y, z}$ are the corresponding components of $\boldsymbol{C}$. Then, condition (32) yields

$\alpha|\boldsymbol{k} \cdot \boldsymbol{b}|\left|C_{y}\right|>\left|k_{x} C_{z}-k_{z} C_{x}\right|$.

The instability occurs only if $\boldsymbol{k}$ has a non-vanishing component perpendicular to the plane $(\boldsymbol{G}, \boldsymbol{C})$. Perturbations are suppressed if $\boldsymbol{k}$ is parallel to this plane and, hence, $C_{y}=0$.
Equation (34) applies only if condition (24) is satisfied and

$\left|k_{x} C_{z}-k_{z} C_{x}\right| \gg \frac{k^{2} \omega_{\eta} \omega_{\kappa}}{\beta G k_{x}}\left(1+\alpha^{2}\right)$.

If inequality (35) is satisfied then we have for unstable perturbations

$\alpha|\boldsymbol{k} \cdot \boldsymbol{b}| C_{y} \gg \frac{k^{2} \omega_{\eta} \omega_{k}}{\beta G k_{x}}\left(1+\alpha^{2}\right)$.

Note the important difference between Eqs. (34) and (35). Condition (34) is linear in $\boldsymbol{k}$, so it can always be satisfied by proper choice of the direction of $\boldsymbol{k}$. On the contrary, inequality (35) is non-linear and yields restrictions on the wavelength of unstable perturbations rather than on their direction.

Consider initially condition (34). This condition is obviously fulfilled if the wavevector is close to the direction determined by

$\frac{k_{x}}{k_{z}}=\frac{C_{x}}{C_{z}} \sim \frac{H}{L}$,

where $L=C_{\perp}^{-1} \sim\left(L_{\mathrm{T}}^{-1}+L_{\mathrm{X}}^{-1}\right)^{-1}, L_{\mathrm{T}} \equiv\left|(\Delta \nabla T / T)_{\perp}^{-1}\right|$, and $L_{\mathrm{X}}=$ $\beta\left|(\nabla Y)_{\perp}\right|^{-1}$ are the temperature and composition lengthscales perpendicular to $\boldsymbol{G}$. One can estimate the angle $\Delta \theta$ around this direction where inequality (34) is still satisfied. Restricting ourselves by linear terms in $\Delta \theta$, we have from Eq. (34)

$\Delta \theta<\alpha \frac{\left|C_{y}\right|}{\left|C_{z}\right|}\left|b_{z}+b_{x} \frac{C_{x}}{C_{z}}\right|$.

Note that the components $C_{x}$ and $C_{y}$ are usually small compared to $C_{z}$,

$\left|C_{z}\right| \sim \frac{1}{H} \gg\left|C_{x}\right| \sim\left|C_{y}\right| \sim \frac{1}{L}$.

Therefore, Eq. (38) is approximately equivalent to

$\Delta \theta<\alpha\left|b_{z}\right| \frac{\left|C_{y}\right|}{\left|C_{z}\right|} \sim \alpha\left|b_{z}\right| \frac{H}{L}$

except the region near the magnetic equator where $b_{z} \approx 0$. This estimate is correct if $\alpha<L / H$, or

$B<2 \times 10^{11} Z L_{5} H_{3}^{-1}\left(1+x^{2 / 3}\right) \mathrm{G}$,

where $L_{5}=L / 10^{5} \mathrm{~cm}$. Note, that for all perturbations with $\boldsymbol{k}$ within angle $\Delta \theta$ we have $k_{x}<k_{z}$. If magnetization is very strong and $\alpha \geq L / H$ then inequality (34) can be satisfied even for perturbations with $k_{x} \sim k_{z}$.

Condition (35) determines the wavelength of unstable perturbations. Estimating $C_{x} \sim 1 / L$, we can transform Eq. (35) into

$\frac{1}{L}>\frac{k}{k_{x}} \frac{\omega_{\eta} \omega_{k}}{\beta G} f(\alpha), \quad f(\alpha)=1+\alpha^{2}$

(we assume that $\boldsymbol{k}$ is not perpendicular to $\boldsymbol{B}$ ). Using the definitions of $\omega_{\eta}$ and $\omega_{\kappa}$ and taking account of Eq. (37), this expression can be rewritten as the condition for the wavelength,

$$
\begin{aligned}
\lambda>\lambda_{\mathrm{cr}} & =2 \pi L^{1 / 4}\left(\frac{k}{k_{x}}\right)^{1 / 4}\left(\frac{\eta_{0} K_{0}}{\beta G}\right)^{1 / 4} f^{1 / 4}(\alpha) \\
& \sim 0.6 f^{1 / 4}\left(\frac{Z^{2} L_{5}^{2}}{H_{3} x^{1 / 3}}\right)^{1 / 4}\left[1+\left(1+x^{2 / 3}\right)^{1 / 2}\right]^{-1 / 4} \mathrm{~cm}
\end{aligned}
$$


we use the electron thermal conductivity by Urpin \& Yakovlev (1980). For applicability of a short wavelength approximation, $\lambda$ and $\lambda_{\text {cr }}$ should be smaller than the vertical lengthscale in the ocean, $H \sim 10-20 \mathrm{~m}$. This condition can in general be satisfied for a wide range of parameters except in the case of a very strong magnetic field. In such field $\alpha \gg 1$, and we have $f \approx \alpha$. Then, critical wavelength $\lambda_{\mathrm{cr}}$ becomes larger than $H$ if

$$
\begin{aligned}
B>B_{\mathrm{d}} \sim & 4 \times 10^{15}\left(\frac{H_{3}}{L_{5}^{2}}\right)^{1 / 2} x^{1 / 6}\left(1+x^{2 / 3}\right) \\
& \times\left[1+\left(1+x^{2 / 3}\right)^{1 / 2}\right]^{1 / 2} \mathrm{G} .
\end{aligned}
$$

Critical field $B_{\mathrm{d}}$ is much stronger than typical magnetic fields of the majority of pulsars.

The growth rate of instability depends on the wavelength and can vary within a wide range. If $\omega_{\mathrm{A}}^{2} \gg \omega_{0}^{2}$ and $\zeta<1$, then $\operatorname{Re} \gamma$ for unstable modes is given by

$\operatorname{Re} \gamma \approx \pm \frac{\omega_{\eta}}{2 \omega_{0}^{2}}\left(\omega_{\mathrm{H}}^{2} \mp \omega_{0}^{2}\right)= \pm \frac{\omega_{\eta}}{2}\left[\frac{\alpha(\boldsymbol{k} \boldsymbol{b}) C_{y}}{k_{x} C_{z}-k_{z} C_{x}} \mp 1\right]$.

The growth rate is maximal if $\boldsymbol{k}$ lies in the plane perpendicular to the plane $(\boldsymbol{G}, \boldsymbol{C})$. Then $C_{x}=0$ and, estimating $C_{y} / C_{z} \sim H / L$, from Eq. (45) we have

$\operatorname{Re} \gamma \approx \frac{\eta_{0} k^{2}}{2} \frac{\alpha(\boldsymbol{k} \boldsymbol{b}) C_{y}}{k_{x} C_{z}} \sim 10^{-3} B_{13} \epsilon^{-1} x^{-1} \lambda_{2}^{-2} \frac{H_{3}}{L_{5}} \mathrm{~s}^{-1}$.

The growth rate increases rapidly with increasing $k$ and reaches its maximum at $\lambda \sim \lambda_{\mathrm{cr}}$. According to Eq. (37), the minimal value of the ratio $\epsilon=k_{x} / k$ is on the order of $H / L$. Substituting this estimate into Eq. (46) and assuming, for example, that $\lambda_{2} \sim$ 1 , we have

$\operatorname{Re} \gamma \sim 0.1 B_{13} x^{-1} \mathrm{~s}^{-1}$.

The growth time for such perturbations is $\sim 10 \mathrm{~s}$ in the layer with $\rho \sim 10^{6} \mathrm{~g} / \mathrm{cm}^{3}$ if $B \sim 10^{13}$ G. Perturbations with a shorter vertical wavelength can grow even faster. For example, perturbations with $\lambda \sim \lambda_{\mathrm{cr}} \sim 10 \mathrm{~cm}$ grow on a timescale of about $0.1 \mathrm{~s}$.

\subsection{Instability of the non-oscillatory modes}

Root (27) describes a couple of non-oscillatory modes that can generally be unstable in the ocean. The instability conditions of these modes are

$A_{0}<0$ or $A_{1}<0$.

If we assume $\omega_{\mathrm{A}}^{2} \gg \omega_{0}^{2}$, which is typical for those neutron stars with $B>3 \times 10^{8} x^{1 / 2} \lambda_{2} H_{3}^{1 / 2} L_{5}^{-1} \mathrm{G}$, then conditions (48) read

$\omega_{\mathrm{X}}^{2}+\omega_{\mathrm{XH}}^{2}>0$,

or

$\omega_{\kappa}\left(\omega_{\mathrm{A}}^{2}-\omega_{\mathrm{X}}^{2}\right)-\omega_{\eta}\left(\omega_{0}^{2}+\omega_{\mathrm{H}}^{2}\right)<0$.

Consider initially the condition (49). If the magnetic field is weak or $\boldsymbol{G}$ and $\nabla X$ are almost parallel (or antiparallel), we have
$\left|\omega_{\mathrm{X}}^{2}\right| \gg\left|\omega_{\mathrm{XH}}^{2}\right|$, and inequality (49) transforms into $\omega_{\mathrm{X}}^{2}>0$. This condition has exactly the same form as the standard criterion of the Ledoux convection. Note, however, the big difference because the standard Ledoux convection is a dynamical instability, whereas the instability considered in this subsection is secular. Using the local cartesian coordinates, we can represent condition $\omega_{\mathrm{X}}^{2}>0$ as

$\nabla_{z} X \equiv \sum_{j=1}^{N-1} \delta_{j} \frac{\mathrm{d} X_{j}}{\mathrm{~d} z}<0$.

As an example, in the simplest case of a mixture of two species with the atomic masses $m_{1}$ and $m_{2}$, Eq. (52) reads $\delta_{1} \mathrm{~d} X_{1} / \mathrm{d} z<$ 0 . If $m_{2}>m_{1}$ then $\delta_{1}=-\left(\partial \rho / \partial X_{1}\right)_{\mathrm{pT}}$ is positive, and the condition of instability is $\mathrm{d} X_{1} / \mathrm{d} z<0$, or $\mathrm{d}\left(n_{1} / n_{2}\right) / \mathrm{d} z<0$ where $n_{1}$ and $n_{2}$ are the number density of species 1 and 2, respectively. Therefore, the instability occurs if the relative number density of a light species increases with depth. Most likely, however, the relative concentration of heavy elements increases with depth and $\omega_{\mathrm{X}}^{2}<0$. Therefore, the instability most likely does not occur in the ocean if $\nabla X \| \boldsymbol{G}$.

If $\nabla X$ and $\boldsymbol{G}$ are not parallel, then the stability properties change drastically. In the general case, inequality (49) yields

$\boldsymbol{G} \cdot \nabla X-\frac{1}{k^{2}}(\boldsymbol{k} \cdot \boldsymbol{G})(\boldsymbol{k} \cdot \nabla X)-\frac{\alpha(\boldsymbol{k} \cdot \boldsymbol{b})}{k^{2}} \boldsymbol{G} \cdot(\boldsymbol{k} \times \nabla X)>0$.

Again using the local cartesian coordinates, we have from this equation

$k_{x} k_{z}\left(\alpha b_{z} \nabla_{y} X+\nabla_{x} X\right)>k_{x}^{2}\left(\nabla_{z} X-\alpha b_{x} \nabla_{y} X\right)$,

where $\nabla_{x, y, z} X$ are cartesian components of $\nabla X$. If the composition varies over the surface, then for any distribution of chemicals, there exists a region in the $\left(k_{x}, k_{z}\right)$-plane where the condition of instability (53) is satisfied. The non-oscillatory modes become unstable if even one of the components $\nabla_{x} X$ or $\nabla_{y} X$ is non-vanishing. If the vertical component of a chemical gradient is large and $\nabla_{z} X>\alpha b_{x} \nabla_{y} X$, then the instability occurs if

$\left|\frac{k_{x}}{k_{z}}\right|<\left|\frac{\alpha b_{z} \nabla_{y} X+\nabla_{x} X}{\nabla_{z} X-\alpha b_{x} \nabla_{y} X}\right|$.

If $\nabla_{z} X<\alpha b_{x} \nabla_{y} X$, then the condition of instability is

$\left|\frac{k_{x}}{k_{z}}\right|>\left|\frac{\alpha b_{z} \nabla_{y} X+\nabla_{x} X}{\nabla_{z} X-\alpha b_{x} \nabla_{y} X}\right|$.

Note that the instability occurs even if the magnetic field is weak and the magnetization parameter $\alpha$ is small, $\alpha \ll 1$. In this case, a surface chemical inhomogeneity can be caused, for example, by accretion from the disc and may have a belt-like structure. If $\alpha \ll 1$, then from Eq. (54) we have

$\left|\frac{k_{x}}{k_{z}}\right|<\left|\frac{\nabla_{x} X}{\nabla_{z} X}\right| \sim \frac{H_{X}}{L_{X}}$,

and the instability arises only for perturbations with the vertical wavelength much shorter than the horizontal one. In a moderate magnetic field with $L_{\mathrm{X}} / H_{\mathrm{X}}>\alpha>1$, inequality (55) yields

$\left|\frac{k_{x}}{k_{z}}\right|<\left|\frac{\alpha b_{z} \nabla_{y} X}{\nabla_{z} X}\right| \sim \alpha \frac{H_{\mathrm{X}}}{L_{\mathrm{X}}}$, 
and the region of unstable wavevectors is more extended than where $\alpha \ll 1$. If $\alpha>L_{\mathrm{X}} / H_{\mathrm{X}}$, then the condition of instability is given by Eq. (56) and reads

$\left|\frac{k_{x}}{k_{z}}\right|>\left|\frac{b_{z}}{b_{x}}\right| \sim 1$.

Note, however, that the condition $\alpha>L_{\mathrm{X}} / H_{\mathrm{X}}$ requires a rather strong magnetic field,

$B>2 \times 10^{11} Z\left(1+x^{2 / 3}\right) \frac{L_{\mathrm{X} 5}}{H_{\mathrm{X} 3}} \mathrm{G}$,

which is impossible in many accreting pulsars $\left(H_{\mathrm{X} 3}=\right.$ $\left.H_{\mathrm{X}} / 10^{3} \mathrm{~cm}, L_{\mathrm{X} 5}=L_{\mathrm{X}} / 10^{5} \mathrm{~cm}\right)$.

Condition (50) generalizes the Chandrasekhar criteria of stationary convection for the case of a chemically inhomogeneous fluid with non-parallel gravity and composition gradient. Since $\omega_{\mathrm{A}}$ is typically much larger than other characteristic frequencies and $\omega_{\kappa} \gg \omega_{\eta}$, it is unlikely that inequality (50) is fulfilled in "standard" neutron stars because these conditions require very low magnetic field and large chemical gradient.

The growth rate of the composition-driven instability $\left(A_{0}<\right.$ 0 ) is always proportional to the gradient of $X$ along the surface. Taking into account that $\omega_{\kappa} \gg \omega_{\eta}$ and assuming that the Alfvén frequency is much greater than any other characteristic frequency, we obtain $\left|A_{1}\right|^{2} \gg\left|A_{0}\right|$. Then, roots 5 and 6 are approximately given by

$\gamma_{5}^{(1)} \approx-\frac{A_{0}}{A_{1}}, \quad \gamma_{6}^{(1)} \approx-A_{1}$.

As it was mentioned, the condition $A_{1}<0$ is most likely not fulfilled in the ocean, which means mode 6 is usually stable. Mode 5, however, can be unstable if $A_{0}<0$. Since $\omega_{\mathrm{A}}^{2} \gg\left|\omega_{0}^{2}\right|$ the growth rate of this mode is

$$
\begin{aligned}
\gamma_{5}^{(1)} & \approx \frac{\omega_{\eta}}{\omega_{\mathrm{A}}^{2}}\left(\omega_{\mathrm{X}}^{2}+\omega_{\mathrm{XH}}^{2}\right) \\
& =\frac{4 \pi \rho G \eta_{0} k_{x}}{(\boldsymbol{k} \cdot \boldsymbol{B})^{2}}\left[\alpha(\boldsymbol{k} \cdot \boldsymbol{B}) \nabla_{y} X-k_{x} \nabla_{z} X+k_{z} \nabla_{x} X\right] .
\end{aligned}
$$

We estimate the growth rate for the case when $\boldsymbol{k}$ lies in the plane perpendicular to the plane $(\boldsymbol{G}, \nabla X)$ and, hence, $(\nabla X)_{x}=0$. Assuming that the magnetic field is not very strong $\left(L_{\mathrm{X}} / H_{\mathrm{X}}>\alpha\right)$ and the wavevector satisfies the condition of instability (54), we obtain from Eq. (61)

$\gamma_{5}^{(1)} \approx \frac{k_{x}}{|\boldsymbol{k} \cdot \boldsymbol{b}|} \frac{G}{\omega_{\mathrm{p} i}}\left|\nabla_{y} X\right| \sim 10^{-8} \frac{A}{Z} \frac{k_{x}}{k_{z}} \frac{g_{14}}{B_{13} L_{\mathrm{X} 5}} \mathrm{~s}^{-1}$,

where $\omega_{\mathrm{p} i}=Z e B / A m_{\mathrm{p}} c$ is the ion gyrofrequency and $g_{14}=$ $\mathrm{g} / 10^{14} \mathrm{~cm} \mathrm{~s}^{-2}$. In contrast to the case of oscillatory modes, the growth rate of a non-oscillatory mode depends on the direction of a wavevector rather than on its value. Instability is faster in a neutron star where the surface inhomogeneities of a chemical composition are stronger. If the magnetic field is not very strong and satisfies the condition $L_{\mathrm{X}} / H_{\mathrm{X}}>\alpha>1$ (or $10^{11} Z \Lambda\left(1+x^{2 / 3}\right) L_{\mathrm{X} 5} / H_{\mathrm{X} 5}>B>10^{9} Z \Lambda\left(1+x^{2 / 3}\right)$ ), then the non-oscillatory mode grows on a relatively short timescale $\sim 1 \mathrm{~d}$ if $L \sim 100 \mathrm{~m}$. Note that the chemical and thermal gradients are very likely to be larger in accreting neutron stars than in isolated ones and one could thus expect more efficient hydrodynamic motions in the ocean of accreting stars.

\section{Discussion}

It turns out that the ocean of neutron stars is subject to different instabilities if the temperature or chemical composition varies over the surface. The main driving forces of instability are the Hall effect and horizontal advection of heat or composition; as a result, the growth rate of instability is proportional to the Hall parameter $\alpha$ and the component of $\nabla T$ or $\nabla X$ along the surface. This point can be clarified by a simple qualitative consideration. Consider the case of oscillatory modes in the magnetized ocean $(\alpha>1)$ with strong chemical inhomogeneities $(|\nabla X| \gg \beta|\Delta \nabla T| / T)$. Then, the amplitude of unstable magnetic perturbations is changed mainly by the Hall effect. For example, amplitude $B_{1 y}$ increases after $\Delta t$ by

$\frac{\Delta B_{1 y}}{\Delta t} \sim \eta_{\wedge}(\boldsymbol{k} \boldsymbol{b})\left(\boldsymbol{k} \times \boldsymbol{B}_{1}\right)_{y} \sim \eta_{\wedge}(\boldsymbol{k} \boldsymbol{b}) \frac{k^{2}}{k_{x}} B_{1 z}$

(using the divergence-free condition (6) for $\boldsymbol{B}_{1}$ ), and small perturbations are marked by the subscript " 1 ". Since oscillatory perturbations are approximately Alfvenic in the ocean, the components of velocity and magnetic field are related by $B_{1 z} \sim B_{1 y}\left(v_{1 z} / v_{1 y}\right)$. For the oscillatory instability, perturbations of the composition are relatively small, $X_{1} \propto\left(\boldsymbol{v}_{1} \cdot \nabla X\right) \approx 0$, then $v_{1 z} / v_{1 y} \sim \nabla X_{y} / \nabla Y_{z}$. Substituting these expressions into Eq. (63), we obtain the growth rate (46). It is seen from this simple consideration that the Hall effect (Eq. (63)) is one of the driving forces of instability which makes qualitatively different from other instabilities in accreting stars.

It is worth noting that we neglect rotation in our stability analysis. Rapid rotation will often change the stability properties of accreting neutron stars. The dispersion Eq. (21) is valid until angular velocity $\Omega$ is small compared to all dynamical frequencies. Since $\omega_{\mathrm{A}}$ is larger than $\omega_{0}$ in many cases of interest, our analysis applies if $\min \left(\omega_{0}, \omega_{\mathrm{H}}\right)>\Omega$. This condition can be written as $\Omega \ll \sqrt{\alpha /(1+\alpha)} \omega_{0}$ or, using expression (33) for $\omega_{0}$, as

$P>2 \times 10^{-3} \frac{L_{5}}{H_{3}} \sqrt{\frac{1+\alpha}{\alpha}} \mathrm{s}$,

where $P$ is the rotation period.

The growth time of both the oscillatory and non-oscillatory instabilities is relatively short, and instabilities are likely to operate operate in a non-linear regime. We can estimate the saturation velocity using the mixing-length model (e.g., Schwarzschild 1958) that assumes that the turnover time of turbulence generated by instability is on the order of the growth time of this instability. Consider, for example, the saturation regime of oscillatory modes. Then, in a turbulent cell with the characteristic vertical lengthscale $\lambda_{z}$, the vertical velocity component in saturation can be estimated as $v_{\mathrm{T} z}\left(\lambda_{z}\right) \sim \lambda_{z} \operatorname{Re} \gamma\left(\lambda_{z}\right)$. Using Eq. (46), we have

$v_{\mathrm{T} z} \sim 0.1 B_{13} x^{-1} \epsilon^{-1} \lambda_{z 2}^{-1} \frac{H_{3}}{L_{5}} \mathrm{~cm} \mathrm{~s}^{-1}$.

Since the instability is anisotropic, the saturated turbulent velocity should be anisotropic as well. From Eq. (4), we can estimate the turbulent velocity along the surface as 
$v_{\mathrm{T} x} \sim v_{\mathrm{T} z}\left(\lambda_{x} / \lambda_{z}\right)$ where $\lambda_{x}$ is the characteristic lengthscale of turbulence along the surface.

Turbulent motions can enhance transport in the ocean both vertically and horizontally. For example, turbulence can efficiently mix the material in the ocean. The coefficient of turbulent diffusion can be estimated as a product of the turbulent lengthscale and velocity in the corresponding direction. Then, we have

$v_{\mathrm{T} z} \sim v_{\mathrm{T} z} \lambda_{z} \sim 10 B_{13} \epsilon^{-1} x^{-1} \frac{H_{3}}{L_{5}} \mathrm{~cm}^{2} \mathrm{~s}^{-1}$

for the coefficient of vertical diffusion, and

$v_{\mathrm{T} x} \sim v_{\mathrm{T} x} \lambda_{x} \sim v_{\mathrm{T} z} \frac{\lambda_{x}^{2}}{\lambda_{z}^{2}} \sim 10 B_{13} \epsilon^{-1} x^{-1} \frac{H_{3}}{L_{5}} \frac{\lambda_{x}^{2}}{\lambda_{z}^{2}} \mathrm{~cm}^{2} \mathrm{~s}^{-1}$

for the coefficient of diffusion along the surface. Assuming that $\epsilon \approx k_{x} / k_{z} \sim H / L$ and $B_{13}=0.1, x=1$, we obtain $v_{\mathrm{T} z} \sim 10^{2} \mathrm{~cm}^{2} \mathrm{~s}^{-1}$. Such diffusion is sufficient for mixing the outer layer with depth $\sim 10 \mathrm{~m}$ on a timescale $\sim$ hours. The rate of vertical turbulent diffusion should be compared to the gravitational sedimentation in the ocean. The coefficient of interspecies diffusion (Brown et al. 2002) reads in our notations as

$\mathcal{D} \sim \frac{4 \times 10^{-3}}{A^{0.5} Z^{0.7} Z_{2}^{0.3}} \frac{T_{7}^{1.2}}{x^{0.6}} \mathrm{~cm}^{2} \mathrm{~s}^{-1}$,

where $Z_{2}$ is the charge of a trace component. This quantity is usually much smaller than $v_{\mathrm{T} z}$. As a result, heavy elements can be transported by turbulence from deep ocean layers to the surface and manifest themselves in spectra of both isolated and accreting neutron stars. Spectral features should be different in spectra of accreting neutron stars with stable and unstable burning. If the accretion rate is high and the burning stable, then spectral features corresponding to CNO group elements could be detected in such stars. For example, these features could be observed in spectra of strongly magnetized accreting neutron stars where the burning is stable and a deep ocean of CNO group elements is formed (see, e.g., Bildsten 1998). On the other hand, if burning is unstable and the star burns $\mathrm{H}$ and $\mathrm{He}$ directly to iron group elements, then mixing caused by instability could increase abundances of these elements in the atmosphere and make the corresponding spectral features detectable.

Turbulent motions can also lead to an efficient horizontal diffusion of the accreted material. The characteristic timescale of turbulent diffusion over the neutron star surface can be estimated as $t_{\mathrm{s}} \sim L^{2} / \nu_{\mathrm{T} x}$. Substituting expression (67) and assuming $\epsilon \sim H / L$, we obtain

$t_{\mathrm{s}} \sim 10^{7} L_{5}^{2} \frac{x}{B_{13}}\left(\frac{\lambda_{z}}{\lambda_{x}}\right)^{2} \mathrm{~s}$.

If $\lambda_{z} / \lambda_{x}=k_{x} / k_{z} \sim H / L$ and $B_{13} \sim 0.1, x \sim 1$ then spreading of the accreted material over the surface proceeds on a timescale of about a few hours.

Apart from mixing, turbulence can also enhance the heat transport. The turbulent thermal diffusivity is comparable to $v_{\mathrm{T} z}$, and the ratio of turbulent and electron thermal diffusivities is

$\frac{\nu_{\mathrm{T}}}{\chi_{(0)}} \sim B_{13} T_{7}^{-1}\left(1+x^{2 / 3}\right) x^{-1} \lambda_{z 2}^{-1}$.
In general, these quantities can have the same order of magnitude. Since $\nabla T$ is subadiabatic, turbulent motions increase the difference between the surface and internal temperature. Note that turbulent heat transport along the surface and vertically should be different for the considered instability with more efficient diffusion along the surface, which can reduce the surface temperature gradient and decrease the contrast between the polar and equatorial temperature.

The non-oscillatory instability, despite being slower than the oscillatory one, can also manifest itself in accreting neutron stars. A sufficiently strong magnetic field can efficiently funnel the accreted material onto the magnetic pole. The characteristic radius of such accretion spots in the polar region is likely to be substantially smaller than the stellar radius, $L \sim 10^{4}-10^{5} \mathrm{~cm}$. In a moderate magnetic field satisfying the inequality $L_{\mathrm{X}} / H_{\mathrm{X}}>\alpha>1$, the growth rate can be estimated if we suppose in Eq. (62) that $k_{x} / k_{z} \sim \alpha H_{\mathrm{X}} / L_{\mathrm{X}}$ (see Eq. (57)). Then,

$\gamma_{5}^{(1)} \sim 10^{-6}\left(1+x^{2 / 3}\right)^{-1} \frac{A}{Z^{2} \Lambda} \cdot \frac{g_{14} H_{\mathrm{X} 3}}{L_{\mathrm{X} 5}^{-2}} \mathrm{~s}^{-1}$.

If $Z$ is not very large and the radius of accretion spots on the surface is $\sim 100 \mathrm{~m}$, then the growth time of instability can reach $10^{4}-10^{5} \mathrm{~s}$. In the non-linear regime, the turbulent velocity induced by this instability is rather small, $\sim 0.1-0.01 L_{\mathrm{X} 5}^{-1} \mathrm{~cm} \mathrm{~s}^{-1}$, if $x \sim g_{14} \sim H_{\mathrm{X} 3} \sim 1$. Slow turbulent motions induced by the non-oscillatory instability in the spot can produce slow variations in the X-ray luminocity of accreting pulsars on the same timescale, which should be longer for accreting neutron stars with a weaker magnetic field.

Note that the criteria and growth rates of instability have been derived under the assumption $c_{\mathrm{s}}>c_{\mathrm{A}}$ or $p>$ $B^{2} / 4 \pi$. Neglecting the quantum effects, we have $p \approx 9.7 \times$ $10^{22} x^{5 / 3}$ dyne $/ \mathrm{cm}^{2}$ in the region where the electron gas is nonrelativistic and $x \leq 1$. Then, our results applies if

$B<B_{\mathrm{cr}}(x) \approx 1.1 \times 10^{12} x^{5 / 6} \mathrm{G}$.

Stability properties of the layers where $c_{\mathrm{A}}>c_{\mathrm{s}}$ (or $B>B_{\mathrm{cr}}$ ) are different from those considered in our study. Generally, perturbations of $T$ and $X$ can be suppressed for modes with $\gamma \sim \omega_{\mathrm{A}}>\omega_{\mathrm{s}}$. As a result, a strong magnetic field in the region where $c_{\mathrm{A}}>c_{\mathrm{s}}$ can reduce the growth rate of instabilities by a factor $\left(c_{\mathrm{A}} / c_{\mathrm{S}}\right)^{2}$. Therefore, the turbulent transport on a short time-scale can be suppressed in these layers, and instability is less efficient in the surface region where $c_{\mathrm{A}}>c_{\mathrm{s}}$ or

$\rho<\rho_{\mathrm{cr}} \approx 2.8 \times 10^{7} B_{13}^{6 / 5} \mathrm{~g} / \mathrm{cm}^{3}$.

A difference in turbulent transport between the regions with $\rho>\rho_{\text {cr }}$ and $\rho<\rho_{\text {cr }}$ could produce a qualitative difference in the burst activity of strongly and weakly magnetized accreting neutron stars. The thermonuclear X-ray burst is triggered when $\mathrm{H} / \mathrm{He}$ burning becomes unstable at the base of the accumulated layer, at a density $\sim 10^{5}-10^{6} \mathrm{~g} / \mathrm{cm}^{3}$ (see, e.g., Bildsten 1998; Cumming \& Bildsten 2000). However, strongly magnetized accreting neutron stars are not observed among X-ray bursters, despite accretion at rates for which the burning of $\mathrm{H} / \mathrm{He}$ should be unstable. This is usually considered as evidence that material is not spreading over the surface on short timescales, so 
the local accretion rate is high enough to stabilize the burning (Joss \& Li 1980). In this model, the burning can be stabilized if spreading is suppressed only in the surface layer above the region with $\rho \sim 10^{5}-10^{6} \mathrm{~g} / \mathrm{cm}^{3}$ (or $x<0.1-1$ ) where the $\mathrm{H} / \mathrm{He}$ burning occurs. One can estimate from Eq. (72) that instability and mixing become less efficient in the layers with $\rho<10^{5}-10^{6} \mathrm{~g} / \mathrm{cm}^{3}$ if

$B>B_{\text {cr }}(x \sim 0.1-1) \sim 10^{11}-10^{12} \mathrm{G}$.

Therefore, one can expect that the accreted material is spreading over the surface layer with $\rho<10^{5}-10^{6} \mathrm{~g} / \mathrm{cm}^{3}$ on a short timescale only in weakly magnetized neutron stars with $B<10^{11}-10^{12} \mathrm{G}$, and the $\mathrm{H} / \mathrm{He}$ burning in such stars should be unstable. On the contrary, in strongly magnetized neutron stars with $B>10^{11}-10^{12} \mathrm{G}$, the spreading over the surface layer requires a longer time, and these neutron stars will not manifest themselves as X-ray bursters. The difference between strongly and weakly magnetized accreting neutron stars will be considered in more detail in a forthcoming paper.

Acknowledgements. This work was supported by the Spanish Ministerio de Ciencia y Tecnologia (grant AYA 2001-3490-C02).

\section{References}

Bildsten, L. 1993, ApJ, 418, L21

Bildsten, L. 1998, in The Many Faces of Neutron Stars, ed. A. Alpar, R. Buccheri, \& J. van Paradijs (Dordrecht: Kluwer)

Bildsten, L., \& Cutler, C. 1995, ApJ, 449, 800

Bildsten, L., Ushomirski, G., \& Cutler, C. 1996, ApJ, 460, 827

Bisnovatyi-Kogan, G., \& Komberg, B. 1974, AZh, 51, 373
Brown, E., \& Bildsten, L. 1998, ApJ, 496, 915

Brown, E., Bildsten, L., \& Chang, P. 2002, ApJ, 574, 920

Chandrasekhar, S. 1961, Hydrodynamic and Hydromagnetic Stability (Oxford: Clarendon)

Cumming, A., \& Bildsten, L. 2000, ApJ, 544, 453

Cumming, A., Zweibel, E., \& Bildsten, L. 2001, ApJ, 557, 958

Fryxell, B., \& Woosley, S. 1982, ApJ, 261, 332

Fujimoto, M. 1988, A\&A, 198, 163

Fujimoto, M. 1993, ApJ, 419, 768

Itoh, N., Kohyama, Y., \& Takeuchi, H. 1987, ApJ, 317, 733

Joss, P., \& Li, F. 1980, ApJ, 238, 287

Landau, L., \& Lifshitz, E. 1971, Fluid Mechanics (London: Pergamon Press)

Lewin, W., van Paradijs, J., \& Taam, R. 1992, Space Sci. Rev., 62, 223

Litwin, C., Brown, E., \& Rosner, R. 2001, ApJ, 553, 788

Lou, Y. 2001, ApJ, 563, L147

Menou, K. 2004, MNRAS, 352, 1381

Miralles, J., Urpin, V., \& Van Riper, K. 1997, ApJ, 480, 358

Parker, E. 1966, ApJ, 145, 811

Potekhin, A., \& Chabrier, G. 2000, Phys. Rev. E, 62, 8554

Rai Choudhuri, A., \& Konar, S. 2002, MNRAS, 332, 933

Schatz, H., Aprachamian, A., Barnard, V., et al. 2001, PRL, 86, 3471

Schatz, H., Bildsten, L., Cumming, A., \& Weischer, M. 1999, ApJ, 524,1014

Schwarzschild, M. 1958, Structure and Evolution of the Stars (Princeton: Princeton Univ. Press)

Slattery, V., Doolen, G., \& De Witt, H. 1980, Phys. Rev. A, 21, 2087

Spitkovsky, A., Levin, Y., \& Ushomirsky, G. 2002, ApJ, 566, 1018

Urpin, V. 2004, A\&A, 421, L5

Urpin, V., \& Gil, Y. 2004. A\&A, 415, 305

Urpin, V., \& Yakovlev, D. 1980, Sov. Astron., 24, 425

Woosley, S., Heger, A., Cumming, A., et al. 2004, ApJS, 151, 75

Zahn, J.-P. 1983, in Astrophysical Processes in Upper Main Sequence Stars, ed. B. Hauk, \& A. Maeder (Pub. Observatoire Genève), 253 